\title{
Wöhl, Stefanie, Elisabeth Springler, Martin Pachel, und Bernhard Zeilinger (Hrsg.) (2020): The State of the European Union. Fault Lines in European Integration Wiesbaden: Springer VS. 274 Seiten. 53,49€
}

\author{
Daniel Mertens
}

Online publiziert: 14. April 2020

(C) Der/die Autor(en) 2020

Im September 2010 hielt José Manuel Barroso die erste „State of the Union“ -Rede im Europäischen Parlament. Die Finanz- und Wirtschaftskrise, so der dama lige EU-Kommissionspräsident, sei die ,größte Herausforderung aller Zei ten“ für die Europäische Union, in der die Solidarität ihrer Mitglieder ,wie n ie zuvor" auf die Probe gestellt wurde - doch der „Test“, befand Barroso, se i bestanden. Doch in den kommenden Jahren sollten noch einige „Tests“ auf die Union zukommen, deren Bestehen die Mehrzahl politikwissenschaftlicher Einsc hätzungen eher in Frage stellt als es zu attestieren. Nun hat auch das an der Fa chhochschule des BFI in Wien verortete Herausgeberteam Wöhl, Springler, Pach el und Zeilinger eine wissenschaftliche Bestandsaufnahme vorgelegt, um aus Sicht der

kritischen politischen Ökonomie die Auswirkungen des seit zehn Jahren anhalt enden Krisenmanagements auf den europäischen Integratio nsprozess zu untersuchen.

Der vorliegende Sammelband identifiziert dabei die Entstehung oder Versc härfung von vier zentralen Bruchstellen (,fault lines“) des Integrationsprozesse s, die auch als Organisationseinheiten des Buchs dienen: (1) ein zunehmender A utoritarismus in der wirtschafts- und demokratiepolitischen Ausgestaltung der In tegration; (2) der Aufstieg der extremen Rechten in den europäischen Parteiensys temen; (3) Prozesse der Finanzialisierung und Militarisierung in der Europäische n Union; und (4) die Zunahme sozialer Exklusion und der Konflikte in der Migration spolitik. Die nur zum Teil in den Kapiteln explizierte übergreifende These lautet , dass das europäische Krisenmanagement der vergangenen Dekade negativ durchs chlägt auf die institutionelle Form, die demokratische Legitimität und die ökon omische Kohärenz der EU. Die einzelnen Kapitel gewichten konzeptionelle Überl egungen und empirische Analyse dazu recht unterschiedlich, aber argumentieren ü

D. Mertens $(\bowtie)$

Institut für Sozialwissenschaften, Universität Osnabrück, Osnabrück, Deutschland

E-Mail: daniel.mertens@uos.de 
berwiegend entlang krisentheoretischer und neo-gramscianischer Traditionslinien. Dadurch eignet sich der Band insbesondere auch für diejenigen, die sich mit den Argumenten einer kritischen Integrationsforschung vertraut machen wollen, wie sie auch im Rahmen der Forschungsgruppe „Staatsprojekt Europa“ verhandelt w erden (vgl. zuletzt und überlappend mit dem vorliegenden

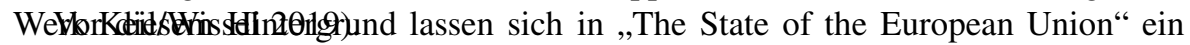
erseits Beiträge finden, die ansonsten unterbelichtete Aspekte des krisenhaften In tegrationsprozesses herausarbeiten, wie zum Beispiel Klatzer und Schlagers Kapi tel zu den geschlechterpolitischen Dimensionen europäischer Reformpolitik. Andere rseits finden sich Beiträge, die neue Perspektiven auf breit diskutierte Phänome ne liefern. So zeigt Salzborns systematisierender Vergleich der extrem-rechten Pa rteien in Europa unter anderem, dass dort sowohl substanzielle Differenzen in stra tegischer als auch inhaltlicher Sicht bestehen; insbesondere über die Frage, ob Re gulierungskompetenzen auf europäisch-zentraler oder regionaler Ebene angesiede lt sein sollten. Das Aufdecken solcher wenig beachteten Konfliktlinien zeigt s ich in Ansätzen auch in den Kapiteln zur Migrationspolitik (z. B. Trauner) und zu glo balen Rivalitäten (Jäger und Roithner). Allein der hypothesengeleitete Beitrag von Zeilinger und Reiner, der den negativen Einfluss der europäischen Austeritätsvorg aben auf sozialpolitische Ausgaben nachweist, fällt hier etwas aus dem Bild; z eigt aber - wie auch Springler und Wöhl - wo kritische und konventionelle politök onぬhinakckdestoweniger erfüllt der BandAdialyisenKlappentext und Einleitung dongedt

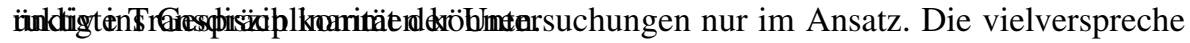
nde Verbindung aus heterodoxer Wirtschaftswissenschaft und Politikwissenschaft, wie sie jüngst auch in der Kombination aus regulationstheoretischen und postkeyn esianischen Analysen von Wachstumsmodellen vorgeschlagen werden, erschöpf $\mathrm{t}$ sich leider in der Einleitung, sodass Verweise auf Keynes, Minsky und Sraff a im Rest des Buchs vergeblich gesucht werden. Auch in der Substanz überrascht es, dass einige augenscheinlich zentrale Projekte des Krisenmanagements nur weni g Aufmerksamkeit erhalten. Die Finanzmarktregulierung samt Banken- und Kapitalma

rktunion wird allein von Bieling und Guntrum - aus recht hoher analytischer Flughöhe - betrachtet; die wie auch immer unzureichenden re-regulativen Projekte i $\mathrm{n}$ der Sozial- und Steuerpolitik bleiben unberücksichtigt. Doch Bruchstellen bieten, vermutlich im Sinne des Herausgeberteams, auch immer die Einfallstore für Geg enbewegungen, weshalb es analytisch hilfreich gewesen wäre, die mit diesen Pro jekten verbundene Widersprüchlichkeit des jüngeren Integrationsprozesses st ärker

denPelsek Kuitikpmokte ungeachtet liegt das Verdienst des Bandes darin, einige span nungsgeladene Dynamiken der vergangenen Dekade, die den Zusammenhalt der EU weiterhin ,testen“, nicht nur aufzuarbeiten, sondern vor allem zusammenzudenke n. Die Notwendigkeit einer solchen kritisch-heterodoxen Perspektive in Forschung u nd Lehre zeigt sich auch im jüngsten integrationspolitischen Moment, der Klimakri se. Es braucht keine weitere „State of the Union“-Rede, um zu erkennen, wie eins chneidend dessen demokratiepolitische und distributive Konsequenzen sein dü rften.

Funding Open Access funding provided by Projekt DEAL. 
Open Access Dieser Artikel wird unter der Creative Commons Namensnennung 4.0 International Li zenz veröffentlicht, welche die Nutzung, Vervielfältigung, Bearbeitung, Verbreitung und Wiederga be in jeglichem Medium und Format erlaubt, sofern Sie den/die ursprünglichen Autor(en) und die Quel le ordnungsgemäß nennen, einen Link zur Creative Commons Lizenz beifügen und angeben, ob $\ddot{A}$ nderungen

vorgenomm en wurden.

Die in diesem Artikel enthaltenen Bilder und sonstiges Drittmaterial unterliegen ebenfalls der genannten Creative Commons Lizenz, sofern sich aus der Abbildungslegende nichts anderes ergibt. Sofern das betref fende Material nicht unter der genannten Creative Commons Lizenz steht und die betreffende Handlu ng nicht nach gesetzlichen Vorschriften erlaubt ist, ist für die oben aufgeführten Weiterverwendunge $\mathrm{n}$ des Materials die Einwilligung des jeweiligen Rechteinhabers einzuho len.

Weitere Details zur Lizenz entnehmen Sie bitte der Lizenzinformation auf http://creativecommons.org/ licenses/by/4.0/deed.de. 